\title{
THE DISCONTINUOUS GALERKIN METHOD WITH DIFFUSION
}

\author{
GERARD R. RICHTER
}

\begin{abstract}
We propose a way of extending the discontinuous Galerkin method from pure hyperbolic equations to convection-dominated equations with an $O(h)$ diffusion term. The resulting method is explicit and can be applied with polynomials of degree $n \geq 1$. The extended method satisfies the same $O\left(h^{n+1 / 2}\right)$ error estimate previously established for the discontinuous Galerkin method as applied to hyperbolic problems. Numerical results are provided.
\end{abstract}

\section{INTRODUCTION}

In this paper we consider a class of constant-coefficient convection-diffusion equations of the form

$$
u_{\alpha}-\sigma_{1} u_{x x}-\sigma_{2} u_{y y}=f, \quad(x, y) \in \Omega \subset R^{2} .
$$

We assume $\Omega$ is a bounded polygon, $\alpha=\left(\alpha_{1}, \alpha_{2}\right)$ is a unit vector, and $\sigma_{1}$ and $\sigma_{2}$ are nonnegative. The term $u_{\alpha}$ denotes the directional derivative $\alpha \cdot \nabla u$. Equation (1.1) may be hyperbolic, parabolic, or elliptic depending on the number of nonzero diffusion coefficients which appear. We are interested in the convection-dominated case where $\sigma_{1}$ and $\sigma_{2}$ are at most $O(h)$ functions of the mesh size $h$ used to discretize (1.1). We assume that Dirichlet data for $u$ is given on the "inflow" portion of the boundary $\Gamma$ of $\Omega$, defined by $\alpha \cdot n<0$, where $n$ is the unit outer normal. In this setting, we will show that the discontinuous Galerkin method can be generalized to (1.1), thus extending its applicability beyond purely hyperbolic problems.

We first describe the discontinuous Galerkin method for the hyperbolic limit of $(1.1)$ :

$$
\left\{\begin{array}{l}
u_{\alpha}=f \text { in } \Omega \\
u \text { given on } \Gamma_{\text {in }}(\Omega) .
\end{array}\right.
$$

Here, $\Gamma_{\text {in }}(\Omega)$ is the "inflow" portion of $\Gamma$, defined by $\alpha \cdot n<0$, where $n$ is the unit outer normal along $\Gamma$. We assume $\Omega$ has been divided into a quasiuniform mesh of triangles of side length $h$ with minimum angle bounded away from zero. For a generic triangle $T$ we denote the space of polynomials of degree $\leq n$ over $T$ by $\mathbf{P}_{n}(T)$. Starting from an interpolant $\left(u^{h}\right)^{-}$of the given initial data on $\Gamma_{\text {in }}(\Omega)$, we seek a (discontinuous) approximation $u^{h}$ which lies

Received June 21, 1990; revised February 19, 1991.

1991 Mathematics Subject Classification. Primary 65N30, 65M15.

Key words and phrases. Finite element, hyperbolic. 
in $\mathbf{P}_{n}(T)$ for each triangle $T$ and satisfies the conditions

$$
\left(u_{\alpha}^{h}, v^{h}\right)-\int_{\Gamma_{\text {in }}(T)}\left[\left(u^{h}\right)^{+}-\left(u^{h}\right)^{-}\right] v^{h} \alpha \cdot n=\left(f, v^{h}\right), \quad \text { all } v^{h} \in \mathbf{P}_{n}(T),
$$

where $(\cdot, \cdot)$ denotes the $L^{2}(T)$ inner product. Here $\left(u^{h}\right)^{-}$and $\left(u^{h}\right)^{+}$are the upstream and downstream limits of $u^{h}$ on $\Gamma_{\text {in }}(T)$, i.e.,

$$
\left(u^{h}\right)^{ \pm}(P) \equiv \lim _{\varepsilon \rightarrow 0+} u^{h}(P \pm \varepsilon \alpha) \text {. }
$$

The boundary integral in (1.3) and all subsequent boundary integrals are taken with respect to arc length.

The first papers dealing with the discontinuous Galerkin method were those of Reed and Hill [7] and Lesaint and Raviart [5]. In the latter, it was shown that the triangles can be ordered explicitly with respect to domain of dependence. Thus, $u^{h}$ as defined by (1.3) can be computed one triangle at a time. In practice, the method is well suited to an adaptive implementation in which the mesh is selected locally as $u^{h}$ evolves. Johnson and Pitkäranta [4] established the error estimate

$$
\left\|u^{h}-u\right\|_{\Omega} \leq C h^{n+1 / 2}\|u\|_{n+1, \Omega}
$$

Here and throughout the paper, $C$ signifies a generic constant, independent of $u$ and the triangulation, and for $D \subset R^{2},\|\cdot\|_{D}$ denotes the $L^{2}(D)$ norm and $\|\cdot\|_{n+1, D}$ the $H^{n+1}(D)$ norm.

We propose the following extension of the discontinuous Galerkin method to $(1.1)$ :

$$
\begin{aligned}
\left(u_{\alpha}^{h}-\sigma_{1} u_{x x}^{h}\right. & \left.-\sigma_{2} u_{y y}^{h}, v^{h}\right)-\int_{\Gamma_{\text {in }}(T)}\left[\left(u^{h}\right)^{+}-\left(u^{h}\right)^{-}\right] v^{h} \alpha \cdot n \\
& +\int_{\Gamma_{\text {in }}^{*}(T)}\left\{\sigma_{1}\left[\left(u_{x}^{h}\right)^{+}-\left(u_{x}^{h}\right)^{-}\right] n_{1}+\sigma_{2}\left[\left(u_{y}^{h}\right)^{+}-\left(u_{y}^{h}\right)^{-}\right] n_{2}\right\} v^{h} \\
= & \left(f, v^{h}\right), \quad \text { all } v^{h} \in \mathbf{P}_{n}(T) .
\end{aligned}
$$

Here $\Gamma_{\text {in }}^{*}(T)$ denotes those sides of $\Gamma_{\text {in }}(T)$ which are not part of $\Gamma_{\text {in }}(\Omega)$ (on $\Gamma_{\text {in }}(\Omega)$ the upstream derivatives $\left(u_{x}^{h}\right)^{-},\left(u_{y}^{h}\right)^{-}$are in general not available). The quantities $n_{1}, n_{2}$ are the $x, y$-components of the unit outer normal to $T$. The boundary integral over $\Gamma_{\text {in }}^{*}(T)$ is analogous to that over $\Gamma_{\text {in }}(T)$ and involves the diffusion, as opposed to convection, portion of (1.1). A similar term was used in $[9,10]$ in extending a continuous finite element scheme for hyperbolic equations to problems with diffusion. As in the case of the hyperbolic problem, $u^{h}$ starts as an interpolant of the given data on $\Gamma_{\text {in }}(\Omega)$, and the triangles are processed in an explicit order relative to the convection term. Thus, any boundary data given for $u$ on $\Gamma_{\text {out }}(\Omega)$ will not be used. The scheme can be viewed as an arbitrarily high-order upwind discretization of both convection and diffusion terms.

Defining $q_{i}=\frac{\sigma_{i}}{h}, i=1,2$, we will prove stability of the method (1.5) under a condition of the form

$$
\max \left\{q_{1}, q_{2}\right\} \leq q^{*}
$$


where $q^{*}>0$. In obtaining this result, we require the triangle sides to be bounded away from the characteristic direction, i.e.,

$$
|\alpha \cdot n| \geq c>0
$$

Under assumptions (1.6) and (1.7), we will derive error estimates of the form

$$
\begin{gathered}
\left|\left(u^{h}-u\right)^{-}\right|_{\Gamma_{\text {out }}(\Omega)}+\left\|u^{h}-u\right\|_{\Omega} \leq C h^{n+1 / 2}\|u\|_{n+1, \Omega}, \\
\left\|\left(u^{h}-u\right)_{\alpha}\right\|_{\Omega, h} \leq C h^{n}\|u\|_{n+1, \Omega}, \\
\sqrt{q_{1}+h}\left\|\left(u^{h}-u\right)_{x}\right\|_{\Omega, h}+\sqrt{q_{2}+h}\left\|\left(u^{h}-u\right)_{y}\right\|_{\Omega, h} \leq C h^{n}\|u\|_{n+1, \Omega} .
\end{gathered}
$$

The notation $|\cdot|$, when used with a subscript as in (1.8), will denote an $L^{2}$ boundary norm. The additional subscript $h$ in (1.9) and (1.10) signifies that the corresponding norms are to be taken in the piecewise sense, e.g., $\left\|\left(u^{h}-u\right)_{\alpha}\right\|_{\Omega, h}=\sqrt{\sum_{T \in \Omega}\left\|\left(u^{h}-u\right)_{\alpha}\right\|_{T}^{2}}$.

The bounds (1.8) and (1.9) are the same as those previously established for the hyperbolic limit [4]. However, (1.10) in conjunction with (1.9) indicates optimal $O\left(h^{n}\right)$ accuracy for $\nabla u^{h}$ if one of the diffusion coefficients $\sigma_{i}$ varies in proportion to $h$ and the corresponding direction is not aligned with $\alpha$. This is an improvement over the hyperbolic estimate of $O\left(h^{n-1 / 2}\right)$ for $\left\|\nabla\left(u-u^{h}\right)\right\|_{\Omega}$, obtained from (1.4) via an inverse inequality. Thus, the addition of an $O(h)$ diffusion term leads to a slightly more favorable analysis.

The above error estimates also hold over any subtriangulation $\Omega^{\prime} \subset \Omega$ for which $\Gamma_{\text {in }}\left(\Omega^{\prime}\right) \subset \Gamma_{\text {in }}(\Omega)$, a result of the explicitness of the method. They can thus be applied over a subregion $\Omega^{\prime}$ which excludes the vicinity of $\Gamma_{\text {out }}(\Omega)$, where a boundary layer typically forms as $\sigma \rightarrow 0$ [11]. Local versions of (1.8)-(1.10), applicable in regions of smoothness, can also be derived using the techniques in [3]. We note, in addition, that although this paper is cast in terms of triangular meshes, its contents apply as well to meshes of triangles and/or rectangles.

The proposed scheme (1.5) is applicable in the convection-dominated regime $\sigma_{1}, \sigma_{2} \leq O(h)$. This is where finite element methods geared to the diffusion term, like Galerkin's method, typically exhibit instabilities. Two other finite element methods for convection-dominated problems are the streamline diffusion method [2, 3] and a continuous finite element method due to Reed and Hill [7] for which analyses are given in $[1,9,10]$. The streamline diffusion method is implicit, while the other two methods are explicit. All three satisfy roughly similar error estimates. The explicit methods have the additional requirement of the nonalignment condition (1.7). (This does not preclude the possibility of applying them to nonlinear problems where $\alpha$ is not known a priori, because their explicitness would allow the mesh to be developed locally, in tandem with the evolving $u^{h}$.)

Our analysis of the method (1.5) appears in the next section. Following that are numerical examples involving piecewise linear approximation of parabolic and elliptic problems of the form (1.1). In an Appendix, we sketch how a slight 
modification of the scheme (1.5) will eliminate the nonalignment requirement (1.7) on the boundary of $\Omega$.

\section{ANALYSIS}

We define $a(\cdot, \cdot)$ to be the form associated with the left-hand side of $(1.5)$ :

$$
\begin{aligned}
a(u, v) \equiv & \left(u_{\alpha}-\sigma_{1} u_{x x}-\sigma_{2} u_{y y}, v\right)-\int_{\Gamma_{\mathrm{in}}(T)}\left(u^{+}-u^{-}\right) v \alpha \cdot n \\
& +\int_{\Gamma_{\mathrm{in}}^{*}(T)}\left[\sigma_{1}\left(u_{x}^{+}-u_{x}^{-}\right) n_{1}+\sigma_{2}\left(u_{y}^{+}-u_{y}^{-}\right) n_{2}\right] v
\end{aligned}
$$

Assuming $v^{-}$is defined on $\Gamma_{\text {in }}(T)$, we obtain upon integrating by parts:

$$
\begin{aligned}
a(u, v)= & -\left(u, v_{\alpha}\right)+\sigma_{1}\left(u_{x}, v_{x}\right)+\sigma_{2}\left(u_{y}, v_{y}\right)+\int_{\Gamma_{\text {out }}(T)} u^{-} v^{-} \alpha \cdot n \\
& +\int_{\Gamma_{\text {in }}(T)}\left[u^{+} v^{+}-\left(u^{+}-u^{-}\right) v^{+}\right] \alpha \cdot n \\
& -\int_{\Gamma_{\text {out }}(T)}\left[\sigma_{1} u_{x}^{-} n_{1}+\sigma_{2} u_{y}^{-} n_{2}\right] v^{-}-\int_{\Gamma_{\text {in }}(T)}\left[\sigma_{1} u_{x}^{+} n_{1}+\sigma_{2} u_{y}^{+} n_{2}\right] v^{+} \\
& +\int_{\Gamma_{\text {in }}^{*}(T)}\left[\sigma_{1}\left(u_{x}^{+}-u_{x}^{-}\right) n_{1}+\sigma_{2}\left(u_{y}^{+}-u_{y}^{-}\right) n_{2}\right] v^{+}
\end{aligned}
$$

This can be expressed in the form

$$
\begin{aligned}
a(u, v)= & -\left(u, v_{\alpha}\right)+\sigma_{1}\left(u_{x}, v_{x}\right)+\sigma_{2}\left(u_{y}, v_{y}\right) \\
& +\oint_{\Gamma(T)} u^{-} v^{-} \alpha \cdot n+\int_{\Gamma_{\text {in }}(T)} u^{-}\left(v^{+}-v^{-}\right) \alpha \cdot n \\
& -\int_{\Gamma_{\text {out }}(T) \cup \Gamma_{\text {in }}^{*}(T)}\left[\sigma_{1} u_{x}^{-} n_{1}+\sigma_{2} u_{y}^{-} n_{2}\right] v^{-} \\
& -\int_{\Gamma_{\text {in }}^{*}(T)}\left[\sigma_{1} u_{x}^{-} n_{1}+\sigma_{2} u_{y}^{-} n_{2}\right]\left(v^{+}-v^{-}\right) \\
& -\int_{\Gamma_{\text {in }}(T)-\Gamma_{\text {in }}^{*}(T)}\left[\sigma_{1} u_{x}^{+} n_{1}+\sigma_{2} u_{y}^{+} n_{2}\right]\left[v^{-}+\left(v^{+}-v^{-}\right)\right] .
\end{aligned}
$$

Note also the following form for the "convection" part of $a(v, v)$ :

$$
\begin{aligned}
\left(v_{\alpha}, v\right) & -\int_{\Gamma_{\text {in }}(T)}\left(v^{+}-v^{-}\right) v^{+} \alpha \cdot n \\
= & \frac{1}{2} \int_{\Gamma_{\text {out }}(T)}\left(v^{-}\right)^{2} \alpha \cdot n-\frac{1}{2} \int_{\Gamma_{\text {in }}(T)}\left[2\left(v^{+}-v^{-}\right) v^{+}-\left(v^{+}\right)^{2}\right] \alpha \cdot n \\
= & \frac{1}{2} \int_{\Gamma_{\text {out }}(T)}\left(v^{-}\right)^{2} \alpha \cdot n-\frac{1}{2} \int_{\Gamma_{\text {in }}(T)}\left[\left(v^{+}-v^{-}\right)^{2}-\left(v^{-}\right)^{2}\right] \alpha \cdot n \\
= & \frac{1}{2} \oint_{\Gamma(T)}\left(v^{-}\right)^{2} \alpha \cdot n+\frac{1}{2} \int_{\Gamma_{\text {in }}(T)}\left(v^{+}-v^{-}\right)^{2}|\alpha \cdot n| .
\end{aligned}
$$


Thus, from (2.2) and (2.3),

$$
\begin{aligned}
a(v, v)= & \frac{1}{2} \oint_{\Gamma(T)}\left(v^{-}\right)^{2} \alpha \cdot n+\frac{1}{2} \int_{\Gamma_{\text {in }}(T)}\left(v^{+}-v^{-}\right)^{2}|\alpha \cdot n|+\sigma_{1}\left\|v_{x}\right\|^{2}+\sigma_{2}\left\|v_{y}\right\|^{2} \\
& -\int_{\Gamma_{\text {out }}(T) \cup \Gamma_{\text {in }}^{*}(T)}\left[\sigma_{1} v_{x}^{-} n_{1}+\sigma_{2} v_{y}^{-} n_{2}\right] v^{-} \\
& -\int_{\Gamma_{\text {in }}^{*}(T)}\left[\sigma_{1} v_{x}^{-} n_{1}+\sigma_{2} v_{y}^{-} n_{2}\right]\left(v^{+}-v^{-}\right) \\
& -\int_{\Gamma_{\text {in }}(T)-\Gamma_{\text {in }}^{*}(T)}\left[\sigma_{1} v_{x}^{+} n_{1}+\sigma_{2} v_{y}^{+} n_{2}\right]\left[v^{-}+\left(v^{+}-v^{-}\right)\right],
\end{aligned}
$$

where $\|\cdot\|$ signifies the $L^{2}(T)$ norm.

Lemma 2.1. Given $\varepsilon>0$, there exist positive constants $q^{*}, \xi_{0}, \xi_{1}, \xi_{2}\left(q^{*}\right.$ depending on $\varepsilon$ ) such that for $\max \left\{q_{1}, q_{2}\right\} \leq q^{*}$

$$
\begin{aligned}
a\left(u^{h}, u^{h}\right) \geq & \frac{1}{2} \oint_{\Gamma(T)}\left[\left(u^{h}\right)^{-}\right]^{2} \alpha \cdot n+\xi_{0}\left|\left(u^{h}\right)^{+}-\left(u^{h}\right)^{-}\right|_{\Gamma_{\text {in }}(T)}^{2} \\
& +\xi_{1} \sigma_{1}\left(\left\|u_{x}^{h}\right\|^{2}-\varepsilon\left\|u_{x}^{h}\right\|_{I(T), h}^{2}\right)+\xi_{2} \sigma_{2}\left(\left\|u_{y}^{h}\right\|^{2}-\varepsilon\left\|u_{y}^{h}\right\|_{I(T), h}^{2}\right) \\
& -\int_{\Gamma_{\text {out }}(T) \cup \Gamma_{\text {in }}^{*}(T)}\left[\sigma_{1}\left(u_{x}^{h}\right)^{-} n_{1}+\sigma_{2}\left(u_{y}^{h}\right)^{-} n_{2}\right]\left(u^{h}\right)^{-} \\
& +C\left|\left(u^{h}\right)^{-}\right|_{\Gamma_{\text {in }}(T)-\Gamma_{\text {in }}^{*}(T)}^{2},
\end{aligned}
$$

where $I(T)$ denotes the triangles, if any, lying on the upstream side of $\Gamma_{\mathrm{in}}^{*}(T)$. Proof. Take $v=u^{h}$ in (2.4). The integral over $\Gamma_{\text {in }}^{*}(T)$ can be bounded via the Schwarz and arithmetic-geometric mean inequalities, and an inverse inequality of the form $\left|\left(v^{h}\right)^{-}\right|_{\Gamma_{\text {in }}^{*}(T)} \leq C h^{-1 / 2}\left\|v^{h}\right\|_{I(T), h}$ :

$$
\begin{aligned}
& \left|\int_{\Gamma_{\text {in }}^{*}(T)}\left[\sigma_{1}\left(u_{x}^{h}\right)^{-} n_{1}+\sigma_{2}\left(u_{y}^{h}\right)^{-} n_{2}\right] \cdot\left[\left(u^{h}\right)^{+}-\left(u^{h}\right)^{-}\right]\right| \\
& \leq C\left[\sigma_{1}\left|\left(u_{x}^{h}\right)^{-}\right|_{\Gamma_{\text {in }}^{*}(T)}+\sigma_{2}\left|\left(u_{y}^{h}\right)^{-}\right|_{\Gamma_{\text {in }}^{*}(T)}\right] \cdot\left|\left(u^{h}\right)^{+}-\left(u^{h}\right)^{-}\right|_{\Gamma_{\text {in }}(T)} \\
& \leq C\left[\sqrt{q_{1} \sigma_{1}}\left\|u_{x}^{h}\right\|_{I(T), h}+\sqrt{q_{2} \sigma_{2}}\left\|u_{y}^{h}\right\|_{I(T), h}\right] \cdot\left|\left(u^{h}\right)^{+}-\left(u^{h}\right)^{-}\right|_{\Gamma_{\text {in }}(T)} \\
& \leq C\left[\sqrt{q_{1}} \sigma_{1}\left\|u_{x}^{h}\right\|_{I(T), h}^{2}+\sqrt{q_{2}} \sigma_{2}\left\|u_{y}^{h}\right\|_{I(T), h}^{2}\right. \\
& \left.+\left(\sqrt{q_{1}}+\sqrt{q_{2}}\right) \cdot\left|\left(u^{h}\right)^{+}-\left(u^{h}\right)^{-}\right|_{\Gamma_{\text {in }}(T)}^{2}\right] .
\end{aligned}
$$

Similarly, the last term of (2.4) with $v=u^{h}$ can be bounded as follows:

$$
\begin{aligned}
& \left|\int_{\Gamma_{\text {in }}(T)-\Gamma_{\text {in }}^{*}(T)}\left[\sigma_{1}\left(u_{x}^{h}\right)^{+} n_{1}+\sigma_{2}\left(u_{y}^{h}\right)^{+} n_{2}\right] \cdot\left\{\left(u^{h}\right)^{-}+\left[\left(u^{h}\right)^{+}-\left(u^{h}\right)^{-}\right]\right\}\right| \\
& \leq C\left\{\sqrt{q_{1}} \sigma_{1}\left\|u_{x}^{h}\right\|^{2}+\sqrt{q_{2}} \sigma_{2}\left\|u_{y}^{h}\right\|^{2}\right. \\
& \left.\quad+\left(\sqrt{q_{1}}+\sqrt{q_{2}}\right)\left[\left|\left(u^{h}\right)^{-}\right|_{\Gamma_{\text {in }}(T)-\Gamma_{\text {in }}^{*}(T)}^{2}+\left|\left(u^{h}\right)^{+}-\left(u^{h}\right)^{-}\right|_{\Gamma_{\text {in }}(T)}^{2}\right]\right\} .
\end{aligned}
$$

The desired result follows.

We now obtain an error estimate for $u^{h}$. We use as a comparison function $u^{I}=P_{n} u$, where $P_{n}$ denotes $L^{2}$ projection into $\mathbf{P}_{n}(T)$. Defining $e^{h}=u^{h}-u^{I}$, 
we have

$$
a\left(e^{h}, e^{h}\right)=a\left(u-u^{I}, e^{h}\right)
$$

From (2.2) and repeated use of the Schwarz and arithmetic-geometric mean inequalities, we obtain for arbitrary $\delta>0$ :

$$
\begin{aligned}
a\left(u-u^{I}, e^{h}\right) \leq & \delta\left(\sigma_{1}\left\|e_{x}^{h}\right\|^{2}+\sigma_{2}\left\|e_{y}^{h}\right\|^{2}+\left|\left(e^{h}\right)^{+}-\left(e^{h}\right)^{-}\right|_{\Gamma_{\text {in }}(T)}^{2}\right) \\
& +C \delta^{-1}\left\{\sigma_{1}\left\|\left(u-u^{I}\right)_{x}\right\|^{2}+\sigma_{2}\left\|\left(u-u^{I}\right)_{y}\right\|^{2}+\left|\left(u-u^{I}\right)^{-}\right|_{\Gamma_{\text {in }}(T)}^{2}\right. \\
& +\sigma_{1}^{2}\left|\left(u-u^{I}\right)_{x}^{-}\right|_{\Gamma_{\text {in }}^{*}(T)}^{2}+\sigma_{2}^{2}\left|\left(u-u^{I}\right)_{y}^{-}\right|_{\Gamma_{\text {in }}^{*}(T)}^{2} \\
& \left.+\sigma_{1}^{2}\left|\left(u-u^{I}\right)_{x}^{+}\right|_{\Gamma_{\text {in }}(T)-\Gamma_{\text {in }}^{*}(T)}^{2}+\sigma_{2}^{2}\left|\left(u-u^{I}\right)_{y}^{+}\right|_{\Gamma_{\text {in }}(T)-\Gamma_{\text {in }}^{*}(T)}^{2}\right\} \\
& +C\left|\left(e^{h}\right)^{-}\right|_{\Gamma_{\text {in }}(T)-\Gamma_{\text {in }}^{*}(T)}^{2}+\oint_{\Gamma(T)}\left(u-u^{I}\right)^{-}\left(e^{h}\right)^{-} \alpha \cdot n \\
& -\int_{\Gamma_{\text {out }}(T) \cup \Gamma_{\text {in }}^{*}(T)}\left[\sigma_{1}\left(u-u^{I}\right)_{x}^{-} n_{1}+\sigma_{2}\left(u-u^{I}\right)_{y}^{-} n_{2}\right]\left(e^{h}\right)^{-} .
\end{aligned}
$$

Using the approximation properties of $u^{I}$ and the fact that $\max \left\{\sigma_{1}, \sigma_{2}\right\} \leq$ $O(h)$, we obtain

$$
\begin{aligned}
a\left(u-u^{I}, e^{h}\right) \leq & \delta\left(\sigma_{1}\left\|e_{x}^{h}\right\|^{2}+\sigma_{2}\left\|e_{y}^{h}\right\|^{2}+\left|\left(e^{h}\right)^{+}-\left(e^{h}\right)^{-}\right|_{\Gamma_{\text {in }}(T)}^{2}\right) \\
& +C \delta^{-1} h^{2 n+1}\|u\|_{n+1, T \cup I(T)}^{2}+C\left|\left(e^{h}\right)^{-}\right|_{\Gamma_{\text {in }}(T)-\Gamma_{\text {in }}^{*}(T)}^{2} \\
& +\oint_{\Gamma(T)}\left(u-u^{I}\right)^{-}\left(e^{h}\right)^{-} \alpha \cdot n \\
& -\int_{\Gamma_{\text {out }}(T) \cup \Gamma_{\text {in }}^{*}(T)}\left[\sigma_{1}\left(u-u^{I}\right)_{x}^{-} n_{1}+\sigma_{2}\left(u-u^{I}\right)_{y}^{-} n_{2}\right]\left(e^{h}\right)^{-} .
\end{aligned}
$$

Taking $\delta$ sufficiently small and applying Lemma 2.1, we obtain

$$
\begin{aligned}
& \frac{1}{2} \oint_{\Gamma(T)}\left[\left(e^{h}\right)^{-}\right]^{2} \alpha \cdot n+\frac{\xi_{0}}{2}\left|\left(e^{h}\right)^{+}-\left(e^{h}\right)^{-}\right|_{\Gamma_{\text {in }}(T)}^{2}+\xi_{1} \sigma_{1}\left(\frac{1}{2}\left\|e_{x}^{h}\right\|^{2}-\varepsilon\left\|e_{x}^{h}\right\|_{I(T), h}^{2}\right) \\
&+\xi_{2} \sigma_{2}\left(\frac{1}{2}\left\|e_{y}^{h}\right\|^{2}-\varepsilon\left\|e_{y}^{h}\right\|_{I(T), h}^{2}\right) \\
& \quad-\int_{\Gamma_{\text {out }}(T) \cup \Gamma_{\text {in }}^{*}(T)}\left[\sigma_{1}\left(e_{x}^{h}\right)^{-} n_{1}+\sigma_{2}\left(e_{y}^{h}\right)^{-} n_{2}\right]\left(e^{h}\right)^{-} \\
& \leq \oint_{\Gamma(T)}\left(u-u^{I}\right)^{-}\left(e^{h}\right)^{-} \alpha \cdot n \\
&-\int_{\Gamma_{\text {out }}(T) \cup \Gamma_{\text {in }}^{*}(T)}\left[\sigma_{1}\left(u-u^{I}\right)_{x}^{-} n_{1}+\sigma_{2}\left(u-u^{I}\right)_{y}^{-} n_{2}\right]\left(e^{h}\right)^{-} \\
&+C\left|\left(e^{h}\right)^{-}\right|_{\Gamma_{\text {in }}(T)-\Gamma_{\text {in }}^{*}(T)}^{2}+C h^{2 n+1}\|u\|_{n+1, T \cup I(T)}^{2} .
\end{aligned}
$$

Taking $\varepsilon$ sufficiently small (if there are at most $m$ triangles on the downstream 
side of $\Gamma_{\text {out }}(T), \varepsilon<\frac{1}{2 m}$ will suffice), and summing over all triangles yields

$$
\begin{aligned}
\frac{1}{2} \oint_{\Gamma(\Omega)} & {\left[\left(e^{h}\right)^{-}\right]^{2} \alpha \cdot n+\xi_{0}^{\prime} \sum_{T \in \Omega}\left|\left(e^{h}\right)^{+}-\left(e^{h}\right)^{-}\right|_{\Gamma_{\text {in }}(T)}^{2} } \\
& +\xi_{1}^{\prime} \sigma_{1}\left\|e_{x}^{h}\right\|_{\Omega, h}^{2}+\xi_{2}^{\prime} \sigma_{2}\left\|e_{y}^{h}\right\|_{\Omega, h}^{2} \\
& -\int_{\Gamma_{\text {out }}(\Omega)}\left[\sigma_{1}\left(e_{x}^{h}\right)^{-} n_{1}+\sigma_{2}\left(e_{y}^{h}\right)^{-} n_{2}\right]\left(e^{h}\right)^{-} \\
\leq & \oint_{\Gamma(\Omega)}\left(u-u^{I}\right)^{-}\left(e^{h}\right)^{-} \alpha \cdot n \\
& -\int_{\Gamma_{\text {out }}(\Omega)}\left[\sigma_{1}\left(u-u^{I}\right)_{x}^{-} n_{1}+\sigma_{2}\left(u-u^{I}\right)_{y}^{-} n_{2}\right]\left(e^{h}\right)^{-} \\
& +C\left(\left|\left(e^{h}\right)^{-}\right|_{\Gamma_{\text {in }}(\Omega)}^{2}+h^{2 n+1}\|u\|_{n+1, \Omega}^{2}\right),
\end{aligned}
$$

where $\xi_{0}^{\prime}, \xi_{1}^{\prime}, \xi_{2}^{\prime}$ are positive constants. Moreover,

$$
\begin{gathered}
\int_{\Gamma_{\text {out }}(\Omega)}\left[\sigma_{1}\left(e_{x}^{h}\right)^{-} n_{1}+\sigma_{2}\left(e_{y}^{h}\right)^{-} n_{2}\right]\left(e^{h}\right)^{-} \\
\leq C\left[\sqrt{q_{1}} \sigma_{1}\left\|e_{x}^{h}\right\|_{\Omega, h}^{2}+\sqrt{q_{2}} \sigma_{2}\left\|e_{y}^{h}\right\|_{\Omega, h}^{2}+\left(\sqrt{q_{1}}+\sqrt{q_{2}}\right)\left|\left(e^{h}\right)^{-}\right|_{\Gamma_{\text {out }}(\Omega)}^{2}\right] \\
\int_{\Gamma_{\text {out }}(\Omega)}\left[\sigma_{1}\left(u-u^{I}\right)_{x}^{-} n_{1}+\sigma_{2}\left(u-u^{I}\right)_{y}^{-} n_{2}\right]\left(e^{h}\right)^{-} \\
\leq C\left[\sqrt{q_{1}} \sigma_{1}\left\|\left(u-u^{I}\right)_{x}\right\|_{\Omega, h}^{2}+\sqrt{q_{2}} \sigma_{2}\left\|\left(u-u^{I}\right)_{y}\right\|_{\Omega, h}^{2}\right. \\
\left.+\left(\sqrt{q_{1}}+\sqrt{q_{2}}\right)\left|\left(e^{h}\right)^{-}\right|_{\Gamma_{\text {out }}(\Omega)}^{2}\right] \\
\leq C\left[\left(\sqrt{q_{1}}+\sqrt{q_{2}}\right)\left|\left(e^{h}\right)^{-}\right|_{\Gamma_{\text {out }}(\Omega)}^{2}+h^{2 n+1}\|u\|_{n+1, \Omega}^{2}\right]
\end{gathered}
$$

and

$$
\begin{aligned}
\frac{1}{2} \oint_{\Gamma(\Omega)} & {\left[\left(e^{h}\right)^{-}\right]^{2} \alpha \cdot n-\oint_{\Gamma(\Omega)}\left(u-u^{I}\right)^{-}\left(e^{h}\right)^{-} \alpha \cdot n } \\
\quad= & \frac{1}{2} \oint_{\Gamma(\Omega)}\left[\left(e^{h}\right)^{-}-\left(u-u^{I}\right)^{-}\right]^{2} \alpha \cdot n+\frac{1}{2} \oint_{\Gamma(\Omega)}\left[\left(u-u^{I}\right)^{-}\right]^{2} \alpha \cdot n \\
\quad \leq & \oint_{\Gamma(\Omega)}\left[\left(u^{h}-u\right)^{-}\right]^{2} \alpha \cdot n+C h^{2 n+1}\|u\|_{n+1, \Omega}^{2} .
\end{aligned}
$$

From (2.7)-(2.10) we infer

$$
\begin{aligned}
& \left|\left(u^{h}-u\right)^{-}\right|_{\Gamma_{\text {out }}(\Omega)}^{2}+\sum_{T \in \Omega}\left|\left(e^{h}\right)^{+}-\left(e^{h}\right)^{-}\right|_{\Gamma_{\text {in }}(T)}^{2}+\sigma_{1}\left\|e_{x}^{h}\right\|_{\Omega, h}^{2}+\sigma_{2}\left\|e_{y}^{h}\right\|_{\Omega, h}^{2} \\
& \quad \leq C\left(\left|\left(u^{h}-u\right)^{-}\right|_{\Gamma_{\text {in }}(\Omega)}^{2}+h^{2 n+1}\|u\|_{n+1, \Omega}^{2}\right) .
\end{aligned}
$$

We shall assume that $\left(u^{h}\right)^{-}$is taken to be a standard interpolant on $\Gamma_{\text {in }}(\Omega)$. We then use the fact that $u^{I}$ is an optimal-order interpolant to conclude:

Theorem 2.1. There exists a constant $q^{*}>0$ such that for $\max \left\{q_{1}, q_{2}\right\} \leq q^{*}$

$$
\left|\left(u^{h}-u\right)^{-}\right|_{\Gamma_{\text {out }}(\Omega)}+\sqrt{\sum_{T \in \Omega}\left|\left(u^{h}\right)^{+}-\left(u^{h}\right)^{-}\right|_{\Gamma_{\text {in }}(T)}^{2}} \leq C h^{n+1 / 2}\|u\|_{n+1, \Omega},
$$




$$
\begin{gathered}
\left\|\left(u^{h}-u\right)_{x}\right\|_{\Omega, h} \leq C q_{1}^{-1 / 2} h^{n}\|u\|_{n+1, \Omega}, \\
\left\|\left(u^{h}-u\right)_{y}\right\|_{\Omega, h} \leq C q_{2}^{-1 / 2} h^{n}\|u\|_{n+1, \Omega} .
\end{gathered}
$$

By taking $v^{h}=u_{\alpha}^{h}$ in (1.5), it can also be shown that

$$
\left\|\left(u^{h}-u\right)_{\alpha}\right\|_{\Omega, h} \leq C h^{n}\|u\|_{n+1, \Omega} .
$$

Note that (2.11) also holds for any subtriangulation $\Omega^{\prime} \subset \Omega$ whose domain of dependence as defined by (1.5) does not include any triangle of $\Omega-\Omega^{\prime}$. This observation, together with the local stability result [4]

$$
\left\|u^{h}\right\|_{T} \leq C\left(\sqrt{h}\left|\left(u^{h}\right)^{-}\right|_{\Gamma_{\text {in }}(T)}+h\|f\|_{T}\right)
$$

for solutions of (1.3), can be used to extend (2.11) to the interior estimate

$$
\left\|u^{h}-u\right\|_{\Omega} \leq C h^{n+1 / 2}\|u\|_{n+1, \Omega} .
$$

\section{COMPUTATIONAL EXAMPLES}

Our first example is the elliptic equation

$$
-\sigma \Delta u+u_{\alpha}=0 \text { in } \Omega,
$$

where $\Omega$, pictured in Figure 3.1, is a trapezoidal domain triangulated via congruent right isosceles triangles. Assuming the angle of inclination of $\alpha$ from the positive $x$-axis is between $\pi / 4$ and $3 \pi / 4, \Gamma_{\text {in }}(\Omega)$ is the $y=0$ portion of $\Gamma(\Omega)$.

A solution of (3.1) that remains globally smooth as $\sigma \rightarrow 0$ is

$$
u^{*}(s, t)=e^{-\lambda s} \sin \omega t, \quad\left\{\begin{array}{l}
s=\alpha_{1} x+\alpha_{2} y, \\
t=\alpha_{2} x-\alpha_{1} y,
\end{array}\right.
$$

where

$$
\omega=\frac{2 \pi}{\alpha_{2}}, \quad \lambda=\frac{\sqrt{1+4 \omega^{2} \sigma^{2}}-1}{2 \sigma} .
$$

As initial data for (3.1) we take

$$
u=u^{*} \text { on } \Gamma_{\text {in }}(\Omega) \text {. }
$$

When $\sigma$ is small, the role of the boundary data on $\Gamma_{\text {out }}(\Omega)$ is, in general, to induce an adjacent outflow boundary layer of width $O(\sigma)$ [11]. Our method uses only the "hyperbolic" boundary data (3.2), and yields an approximation

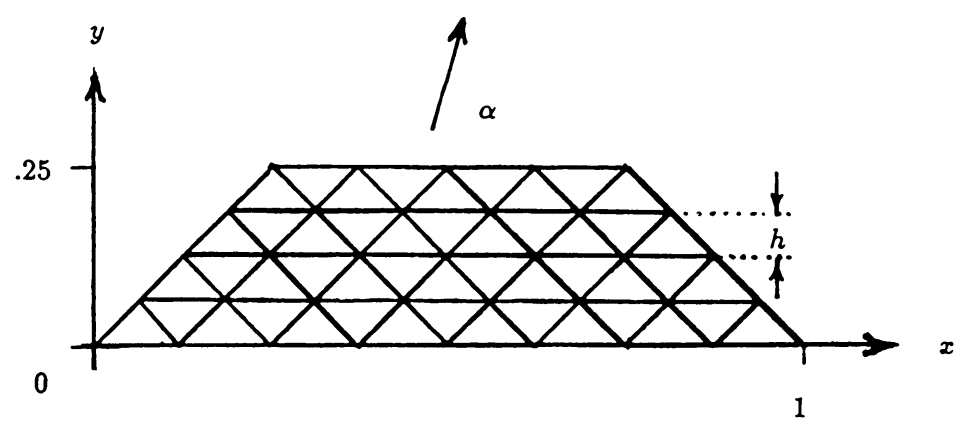

FigURE 3.1 
TABLE 3.1

\begin{tabular}{rcc}
\hline$h^{-1}$ & $E_{0}$ & ratio \\
\hline 16 & .121 & - \\
32 & $.682(-1)$ & 1.78 \\
64 & $.284(-1)$ & 2.40 \\
128 & $.937(-2)$ & 3.03 \\
256 & $.270(-2)$ & 3.47 \\
512 & $.727(-3)$ & 3.72 \\
1024 & $.189(-3)$ & 3.86 \\
2048 & $.480(-4)$ & 3.93 \\
\hline
\end{tabular}

$u^{h}$ that does not have this boundary layer. In the present case, Theorem 2.1 implies that $u^{h} \rightarrow u^{*}$ as $h \rightarrow 0$ provided $\sigma / h \leq q^{*}$.

Table 3.1 corresponds to piecewise linear approximation of (3.1), (3.2) with $\alpha$ inclined at an angle $\pi / 3$ from the horizontal and $\sigma / h=1$, where $h$ is the triangle height. Weighted $L^{2}$ errors

$$
E_{0} \equiv \sqrt{\int_{\Gamma_{\text {out }}(\Omega)}\left[u^{*}-\left(u^{h}\right)^{-}\right]^{2}|\alpha \cdot n|}
$$

are given, as well as ratios of successive such quantities as $h$ was continually halved. The results indicate an optimal $O\left(h^{2}\right)$ rate of convergence, which is better than the theoretical prediction in (2.11). This pattern is typical of the discontinuous Galerkin method as applied to the hyperbolic problem (1.2) in the presence of the condition (1.7) $[6,8]$.

As our second example, we take the parabolic equation

$$
u_{\alpha}-\sigma u_{x x}=0 \text { in } \Omega \text {, }
$$

where $\Omega$ and its triangulation, as well as the characteristic direction $\alpha$, are the same as in the previous example. We take as initial data for (3.3)

$$
u(x, 0)=\sin x \text { on } \Gamma_{\text {in }}(\Omega) .
$$

The problem defined by (3.3), (3.4) has the globally smooth (as $\sigma \rightarrow 0$ ) solution

$$
u^{*}(x, y)=\exp \left(-\frac{\sigma y}{\alpha_{2}}\right) \sin \left(x-\frac{\alpha_{1}}{\alpha_{2}} y\right),
$$

which $u^{h}$ will approximate.

The results in Table 3.2 correspond to piecewise linear approximation and $\sigma / h=1$. The optimal $O\left(h^{2}\right)$ convergence rate is again observed. 
TABLE 3.2

\begin{tabular}{rcc}
\hline$h^{-1}$ & $E_{0}$ & ratio \\
\hline 16 & $.164(-2)$ & - \\
32 & $.522(-3)$ & 3.14 \\
64 & $.148(-3)$ & 3.54 \\
128 & $.391(-4)$ & 3.77 \\
256 & $.101(-4)$ & 3.89 \\
512 & $.255(-5)$ & 3.94 \\
1024 & $.643(-6)$ & 3.97 \\
2048 & $.161(-6)$ & 3.99 \\
\hline
\end{tabular}

\section{APPENDIX}

The nonalignment condition (1.7) is an important assumption of our analysis. It cannot not be satisfied, however, if the boundary $\Gamma$ of $\Omega$ contains a portion which is tangent or nearly tangent to $\alpha$. Here we sketch a slight modification of the basic scheme which eliminates the need for the nonalignment restriction on $\Gamma$.

Let us consider a triangle $T$, as depicted in Figure 4.1, which has a side $\Gamma_{1} \subset \Gamma$ where (1.7) is violated. We shall assume Dirichlet data for $u$ is available on $\Gamma_{1}$ whether or not it is an inflow side of $T$.

For such a triangle, we append to the left-hand side of (1.5)

$$
-\int_{\Gamma_{1}}\left[\left(u^{h}\right)^{\mathrm{ext}}-\left(u^{h}\right)^{\mathrm{int}}\right]\left[\sigma_{1}\left(v_{x}^{h}\right) n_{1}+\sigma_{2}\left(v_{y}^{h}\right) n_{2}\right],
$$

where $\left(u^{h}\right)^{\text {ext }}$ is an optimal-order interpolant of the given Dirichlet data on $\Gamma_{1}$ and $\left(u^{h}\right)^{\text {int }}$ is the limit of $u^{h}$ as $\Gamma_{1}$ is approached from the interior of $T$. (If $\Gamma_{1}$ is an inflow side of $T$, then $\left(u^{h}\right)^{\text {ext }}=\left(u^{h}\right)^{-}$and $\left(u^{h}\right)^{\text {int }}=\left(u^{h}\right)^{+}$; if $\Gamma_{1}$ is an outflow side, the situation is reversed.) With the corresponding modification made to the bilinear form $a(u, v)$ in $\S 2,(2.2),(2.4)$, and (2.5)

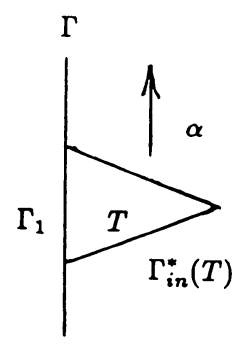

FIGURE 4.1 
become, respectively,

$$
\begin{aligned}
& a(u, v)=-\left(u, v_{\alpha}\right)+\sigma_{1}\left(u_{x}, v_{x}\right)+\sigma_{2}\left(u_{y}, v_{y}\right) \\
& +\oint_{\Gamma(T)} u^{-} v^{-} \alpha \cdot n+\int_{\Gamma_{\mathrm{in}}(T)} u^{-}\left(v^{+}-v^{-}\right) \alpha \cdot n \\
& -\int_{\Gamma(T)-\Gamma_{1}}\left[\sigma_{1} u_{x}^{-} n_{1}+\sigma_{2} u_{y}^{-} n_{2}\right] v^{-} \\
& -\int_{\Gamma_{\mathrm{in}}^{*}(T)}\left[\sigma_{1} u_{x}^{-} n_{1}+\sigma_{2} u_{y}^{-} n_{2}\right]\left(v^{+}-v^{-}\right) \\
& -\int_{\Gamma_{1}}\left\{\sigma_{1}\left[u_{x}^{\mathrm{int}} v^{\mathrm{int}}+\left(u^{\mathrm{ext}}-u^{\mathrm{int}}\right) v_{x}^{\mathrm{int}}\right] n_{1}\right. \\
& \left.+\sigma_{2}\left[u_{y}^{\text {int }} v^{\text {int }}+\left(u^{\text {ext }}-u^{\text {int }}\right) v_{y}^{\text {int }}\right] n_{2}\right\} \\
& a(v, v)=\frac{1}{2} \oint_{\Gamma(T)}\left(v^{-}\right)^{2} \alpha \cdot n+\frac{1}{2} \int_{\Gamma_{\mathrm{in}}(T)}\left(v^{+}-v^{-}\right)^{2}|\alpha \cdot n|+\sigma_{1}\left\|v_{x}\right\|^{2}+\sigma_{2}\left\|v_{y}\right\|^{2} \\
& -\int_{\Gamma(T)-\Gamma_{1}}\left[\sigma_{1} v_{x}^{-} n_{1}+\sigma_{2} v_{y}^{-} n_{2}\right] v^{-} \\
& -\int_{\Gamma_{\text {in }}^{*}(T)}\left[\sigma_{1} v_{x}^{-} n_{1}+\sigma_{2} v_{y}^{-} n_{2}\right]\left(v^{+}-v^{-}\right) \\
& -\int_{\Gamma_{1}}\left[\sigma_{1} v_{x}^{\mathrm{int}} n_{1}+\sigma_{2} v_{y}^{\mathrm{int}} n_{2}\right] v^{\mathrm{ext}},
\end{aligned}
$$

and

$$
\begin{aligned}
a\left(u^{h}, u^{h}\right) \geq & \frac{1}{2} \oint_{\Gamma(T)}\left[\left(u^{h}\right)^{-}\right]^{2} \alpha \cdot n+\xi_{0} \int_{\Gamma_{\text {in }}(T)}\left[\left(u^{h}\right)^{+}-\left(u^{h}\right)^{-}\right]^{2}|\alpha \cdot n| \\
& +\xi_{1} \sigma_{1}\left(\left\|u_{x}^{h}\right\|^{2}-\varepsilon\left\|u_{x}^{h}\right\|_{I(T), h}^{2}\right)+\xi_{2} \sigma_{2}\left(\left\|u_{y}^{h}\right\|^{2}-\varepsilon\left\|u_{y}^{h}\right\|_{I(T), h}^{2}\right) \\
& -\int_{\Gamma(T)-\Gamma_{1}}\left[\sigma_{1}\left(u_{x}^{h}\right)^{-} n_{1}+\sigma_{2}\left(u_{y}^{h}\right)^{-} n_{2}\right]\left(u^{h}\right)^{-}+C\left|\left(u^{h}\right)^{\text {ext }}\right|_{\Gamma_{1}}^{2}
\end{aligned}
$$

In obtaining error estimates, we must bound an additional term arising from $a\left(u-u^{I}, e^{h}\right)$ :

$$
\begin{aligned}
\chi & \equiv\left|\int_{\Gamma_{1}}\left[\sigma_{1}\left(u-u^{I}\right)_{x}^{\text {int }} n_{1}+\sigma_{2}\left(u-u^{I}\right)_{y}^{\text {int }} n_{2}\right]\left(e^{h}\right)^{\text {int }}\right| \\
& \leq C\left(\sigma_{1}+\sigma_{2}\right)\left|\left(e^{h}\right)^{\text {int }}\right|_{\Gamma_{1}} h^{n-1 / 2}\|u\|_{n+1} .
\end{aligned}
$$

Note that

$$
\begin{aligned}
& \sigma_{1}\left|\left(e^{h}\right)^{\mathrm{int}}\right|_{\Gamma_{1}} \leq C \sigma_{1}\left\{\left|\left(e^{h}\right)^{-}\right| \Gamma(T)-\Gamma_{1}+\left|\left(e^{h}\right)^{+}-\left(e^{h}\right)^{-}\right|_{\Gamma_{\text {in }}^{*}(T)}+\sqrt{h}\left\|e_{x}^{h}\right\|\right\} \\
& \leq C h\left\{\left(\oint_{\Gamma(T)}\left|\left(e^{h}\right)^{-}\right|^{2}|\alpha \cdot n|+\int_{\Gamma_{\text {in }}(T)}\left|\left(e^{h}\right)^{+}-\left(e^{h}\right)^{-}\right|^{2}|\alpha \cdot n|\right)^{1 / 2}\right. \\
&\left.+\sqrt{\sigma_{1}}\left\|e_{x}^{h}\right\|\right\},
\end{aligned}
$$


since $\sigma_{1}=O(h)$. We also have an analogous bound for $\sigma_{2}\left|\left(e^{h}\right)^{\text {int }}\right| \Gamma_{1}$ involving $\left\|e_{y}^{h}\right\|$. The arithmetic-geometric mean inequality then gives

$$
\begin{aligned}
\chi \leq & \delta h\left\{\oint_{\Gamma(T)}\left[\left(e^{h}\right)^{-}\right]^{2}|\alpha \cdot n|+\int_{\Gamma_{\text {in }}(T)}\left[\left(e^{h}\right)^{+}-\left(e^{h}\right)^{-}\right]^{2}|\alpha \cdot n|+\sigma_{1}\left\|e_{x}^{h}\right\|^{2}+\sigma_{2}\left\|e_{y}^{h}\right\|^{2}\right\} \\
& +C \delta^{-1} h^{2 n}\|u\|_{n+1}^{2}
\end{aligned}
$$

for arbitrary $\delta>0$. Thus, from $a\left(e^{h}, e^{h}\right)=a\left(u-u^{I}, e^{h}\right)$, we can obtain a bound of the form

$$
\begin{aligned}
& \left(\frac{1}{2}+h\right) \int_{\Gamma_{\text {out }}(\Omega)}\left[\left(e^{h}\right)^{-}\right]^{2}|\alpha \cdot n|+\frac{\xi_{0}}{2} \int_{\Gamma_{\text {in }}(T)}\left[\left(e^{h}\right)^{+}-\left(e^{h}\right)^{-}\right]^{2}|\alpha \cdot n| \\
& +\xi_{1} \sigma_{1}\left(\frac{1}{2}\left\|e_{x}^{h}\right\|^{2}-\varepsilon\left\|e_{x}^{h}\right\|_{I(T), h}^{2}\right)+\xi_{2} \sigma_{2}\left(\frac{1}{2}\left\|e_{y}^{h}\right\|^{2}-\varepsilon\left\|e_{y}^{h}\right\|_{I(T), h}^{2}\right) \\
& \quad-\int_{\Gamma(T)-\Gamma_{1}}\left[\sigma_{1}\left(e_{x}^{h}\right)^{-} n_{1}+\sigma_{2}\left(e_{y}^{h}\right)^{-} n_{2}\right]\left(e^{h}\right)^{-} \\
& \leq \quad\left(\frac{1}{2}-h\right) \int_{\Gamma_{\text {in }}(\Omega)}\left[\left(e^{h}\right)^{-}\right]^{2}|\alpha \cdot n|+\oint_{\Gamma(T)}\left(u-u^{I}\right)^{-}\left(e^{h}\right)^{-} \alpha \cdot n \\
& \quad-\int_{\Gamma(T)-\Gamma_{1}}\left[\sigma_{1}\left(u-u^{I}\right)_{x}^{-} n_{1}+\sigma_{2}\left(u-u^{I}\right)_{y}^{-} n_{2}\right]\left(e^{h}\right)^{-} \\
& \quad+C\left|\left(e^{h}\right)^{\operatorname{ext}}\right|_{\Gamma_{1}}^{2}+C h^{2 n+1}\left(\|u\|_{n+1, T \cup I(T)}^{2}+h^{-1}\|u\|_{n+1}^{2}\right) .
\end{aligned}
$$

Let $\Omega^{\dagger}$ denote the union of those boundary triangles for which the special prescription is used. For a given $\Omega$, our mesh assumptions (quasi-uniformity, minimum angle condition) imply that $\Omega^{\dagger}$ consists of at most $O\left(h^{-1}\right)$ triangles. The above estimate, together with that of $\S 2$ for triangles in $\Omega-\Omega^{\dagger}$, can be summed in a layer-by-layer fashion, as in [1]. The result will be a set of analogs of $(1.8)-(1.10)$, in which $\left|\left(u^{h}-u\right)^{-}\right|_{\Gamma_{\text {out }}(\Omega)}$ is replaced by $\left\{\int_{\Gamma_{\text {out }}(\Omega)}\left[\left(u^{h}-u\right)^{-}\right]^{2}|\alpha \cdot n|\right\}^{1 / 2}$ and $\|u\|_{n+1, \Omega}$ is replaced by $\|u\|_{n+1, \Omega}+$ $h^{-1 / 2}\|u\|_{n+1, \Omega^{\dagger}}$. Note that the area of $\Omega^{\dagger}$ is of order $h$. Thus, if $u$ is sufficiently smooth in $\Omega^{\dagger}$ (e.g., $u \in W^{n+1, \infty}\left(\Omega^{\dagger}\right)$ ), we retain the same order of accuracy as given by the estimates in $\S 2$.

\section{BIBLIOGRAPHY}

1. R. S. Falk and G. R. Richter, Analysis of a continuous finite element method for hyperbolic equations, SIAM J. Numer. Anal. 24 (1987), 257-278.

2. T. J. R. Hughes and A. Brooks, A multidimensional upwind scheme with no crosswind diffusion, Finite Element Methods for Convection Dominated Flows (T. J. R. Hughes, ed.), AMD (ASME) 34 (1979).

3. C. Johnson, U. Nävert, and J. Pitkäranta, Finite element methods for linear hyperbolic problems, Comput. Methods Appl. Mech. Engrg. 45 (1984), 285-312.

4. C. Johnson and J. Pitkäranta, An analysis of the discontinuous Galerkin method for a scalar hyperbolic equation, Math. Comp. 46 (1986), 1-26.

5. P. Lesaint and P. A. Raviart, On a finite element method for solving the neutron transport equation, Mathematical Aspects of Finite Elements in Partial Differential Equations, (C. deBoor, ed.), Academic Press, 1974, pp. 89-123.

6. T. E. Peterson, A note on the convergence of the discontinuous Galerkin method for a scalar hyperbolic equation, preprint. 
7. W. H. Reed and T. R. Hill, Triangular mesh methods for the neutron transport equation, Los Alamos Scientific Laboratory Report LA-UR-73-479, 1973.

8. G. R. Richter, An optimal-order error estimate for the discontinuous Galerkin method, Math. Comp. 50 (1988), 75-88.

9. _ A finite element method for time-dependent convection-diffusion equations, Math. Comp. 54 (1990), 81-106.

10. __ An explicit finite element method for convection-dominated steady state convectiondiffusion equations, SIAM J. Numer. Anal. 28 (1991), 744-759.

11. M. I. Vishik and L. A. Lyusternik, Regular degeneration and boundary layer for linear differential equations with a small parameter, Uspekhi Mat. Nauk 12 (1957), 3-122; English transl. in Amer. Math. Soc. Transl. (2) 20 (1962), 239-364.

Department of Computer Sciences, Rutgers University, New Brunswick, New Jersey 08903 